\title{
MUSES PROJECT OF RIKEN RI BEAM FACTORY
}

\author{
T. Katayama, S. Watanabe \\ Center for Nuclear Study, School of Science, Univ. of Tokyo, Tanashi, Tokyo188-0002, Japan \\ Y. Batygin, N. Inabe, K. Ohtomo, T. Ohkawa, M. Takanaka, T. Tanabe, \\ M. Wakasugi, I. Watanabe, Y. Yano and K. Yoshida \\ Institute of Physical and Chemical Research(RIKEN), Wako, Saitama 351-01, Japan
}

\section{Abstract}

At RIKEN, the construction of Radio Isotope Beam factory, RIBF has been strated from the fiscal year 1998. In this project, the superconducting ring cyclotron with $\mathrm{K}$ number of 2500 will be constructed within 4 years, and the MUSES, Multi-USe Experimental Storage rings, is scheduled to start the construction just after the completion of cyclotron system. In the present paper, the outline of MUSES project is presented, while the details are given elsewhere[1,2] and in this conference.

\section{INTRUDUCTION}

A new type of experimental facility, MUSES (Fig.1) is planned for the RIBF. It consists of an Accumulator Cooler Ring(ACR), a Booster Synchrotron Ring(BSR) with an injector electron linac and Double Storage Rings(DSR). MUSES will be installed downstream from the Superconducting Ring Cycrotron(SRC) and RI separator. The ACR functions for the accumulation and cooling of RI beams and also for experiments such as internal target nuclear physics, and atomic and molecular physics with cooler electron beams. The BSR works solely for the acceleration of ion and electron beams. The DSR permits various types of unique colliding experiments; ion-ion merging or collision; collisions of electron and ion beams, and collisions of RI beams with high brilliant X-ray from an undulator. In the present paper, the basic concept of MUSES accelerator will be described, emphasizing the case of electron-RI collisions at DSR.

\section{RI BEAMS AND ACCUMULATOR COOLER RING}

\subsection{Accumulation Scheme}

Beams extracted from a SRC irradiate a target and are converted to RI beams through the fragmentation process. RI beams produced are the mixture of various nuclei, therefore, they will be purified by means of momentumand charge state selection at Big-RIPS and will be accumulated in the ACR with use of the method of multiturn injection and RF stacking. Momentum cooling will be continuouslly applied for the stacked beam during the RF stacking process. [3] After the accumulation of RI beams in the ACR, of which the intensity is determined by the balance of the intrinsic life time of unstable nuclei and the injection rate, they will be fast extracted from the ACR and injected into the BSR. In the BSR, beams will be accelerated to the required energy. These beams will be injected into the one of the DSR rings. Typical experiment investigated at the DSR is the collision of RI beams with electron beam to study the form factor of the unstable nuclei. In the experiment, another ring of the DSR will be filled with high current $\sim 500 \mathrm{~mA}$ electron beam with energy of up to $2.5 \mathrm{GeV}$.

Typical RI beam characteristics are estimated as follows: the momentum spread is $0.5 \%$, the phase width relative to RF frequency is 5 degrees, and the transverse emittance is $4.5 \pi \mathrm{mm}$ mrad in both horizontal and vertcal directions. This beam is transported from the target point to the injection point of the ACR along the length of 70 $\mathrm{m}$. At the end point of transport line, a debuncher system with $3.8 \mathrm{MV}$ voltage and harmonics number 6 , will be installed to reduce the momentum spread to $0.15 \%$. In Table 1, main parameters of the ACR are tabulated.

\section{Table 1 Main Parameters of Accumulator Ring}

\begin{tabular}{lr} 
Circumference $C(\mathrm{~m})$ & 168.4836 \\
Max. Magnetic Rigidity $B \rho(\mathrm{Tm})$ & 7.244 \\
Max. Beam Energy $T_{\text {max }}(\mathrm{MeV} / \mathrm{u})$ & $500(\mathrm{q} / \mathrm{A}=0.5)$ \\
Transition Gamma $\gamma_{t}$ & 4.987 \\
Betatron Tune Values $Q_{x} / Q_{y}$ & $4.555 / 3.540$ \\
Natural Chromaticity $Q^{\prime} / Q_{y}^{-}$ & $-5.058 /-6.571$ \\
Beam Accepetance $(\mathrm{mm}-\mathrm{mrad})$ & $125 \pi$ \\
Momentum Accepetance $\Delta P / P(\%)$ & 2.5 \\
Injection Method & \\
\multicolumn{2}{c}{ Multiturn injection +RF stacking } \\
Beam Cooling Method
\end{tabular}

Stochastic+Electron Cooling

\subsection{Cooling of Unstable Nucleus Beam}

Both electron cooling[4] and stochastic cooling were studied for the momentum cooling of particles. It is turned out that the stochastic cooling is much faster than the electron cooling for the present case. Electron cooler 
with $3 \mathrm{~m}$ length and current density of $0.5 \mathrm{~A} / \mathrm{cm}^{2}$ gives the cooling time of $380 \mathrm{sec}$ for ${ }^{6} \mathrm{He}$ and $0.42 \mathrm{sec}$ for ${ }^{232} \mathrm{U}$, whereas the stochastic cooling with $10 \mathrm{~kW}$ and a band width of $2 \mathrm{GHz}$ feed back system gives $0.20 \mathrm{~s}$ and $5.0 \mathrm{~ms}$, respectively. This is due to the following property of RI beam. The intensity is rather weak, and momentum and emittance spread are large.

\subsection{Accumulation of Unstable Nuclei}

The accumulation of RI beam is performed by using the RF stacking technique. RI beam is injected into the ACR by means of multiturn injection with the time interval of the cooling time. The beam accumulated in the ACR is decaying with its own intrinsic life time. The maximum number of the nuclei stored in the ACR is determined by the balance of supply rate and the decay rate.

The space charge limit of the ACR is also considered in the estimation of the maximum number of the nuclei stored in the ACR. However the space charge limit is important only for the high production rate nuclei such as stable nuclei and their neighbors.

The accumulated RI beam will be fast extracted from the ACR and injected into the BSR. In the BSR, beams will be accelerated to the energy required for the experiment within $1 \mathrm{sec}$, and then will be injected into the one ring of the DSR to collide with electrons stored in another ring. Since RI beams are bunched by 46 pulses in the DSR, the maximum number of unstable nuclei in a bunch is obtained by dividing the maximum stored intensity in the ACR by 46.

\section{DOUBLE STORAGE RINGS}

The DSR consists of vertically stacked two rings of the similar specification. Each lattice structure takes the form of a racetrack to accommodate two long straight sections. These straight sections of one ring vertically intersect those of the other ring at two colliding points. Two colliding long straight sections are prepared, one is for the collision between RI beam and electron beam with a collision angle $20 \mathrm{mrad}$ (colliding section), and the other is for the merging of ion beams with merging angle 170 mrad (merging section). The RF cavities and beam injection devices are placed at the long straight sections. Two short straight sections will be used for electron coolers to suppress the beam instabilities and to make a short-bunch ion-beams[5].

For the case of electron-RI collision, the lattice of electron ring was designed so that the emittance of electron beam is $10^{-6} \pi$ mrad from the point of view of luminosity and beam-beam effect. Parameters of the DSR for this case are summarized in Table 2.

Another usage of electron beam is to produce high brilliant X-ray and it requires to have small emittance $\left(10^{-8}\right.$ $\pi$ mrad). It is achieved by adopting Double Bend Achromart (DBA) system in the arc section.

\section{$\underline{\text { Table } 2 \text { Parameters of Double Storage Rings }}$}

$\begin{array}{lr}\text { Circumference } C(\mathrm{~m}) & 269.568 \\ \text { Max } B \rho(\mathrm{Tm}) & 14.60 \\ \text { Average Radius } R(\mathrm{~m}) & 42.904 \\ \text { Radius of Curvature } \rho(\mathrm{m}) & 9.733 \\ \text { Max. Stored Beam Energy } & \\ \quad \text { Proton }(\mathrm{GeV}) & 3.55 \\ \quad \text { Ion }(\mathrm{q} / \mathrm{A}=0.5) & 1.45 \\ \quad \text { Electron } & 2.5\end{array}$

\begin{tabular}{lrc} 
Operation mode & Electron & \multicolumn{1}{c}{ Ion } \\
Betatron Tune $Q_{x} / Q_{y}$ & $6.754 / 8.163$ & $6.235 / 5.018$ \\
Chromaticity $Q_{x} / Q_{y}$ & $-37.7 /-90.7$ & $-62.7 /-47.6$ \\
Transition $\gamma$ & 4.857 & 5.071 \\
Max $\beta$ values $\beta_{x} / \beta_{y}(\mathrm{~m})$ & $113 / 750$ & $1223 / 970$ \\
$\beta$ values at Interation point & \\
$\beta_{*}^{*} / \beta_{y}{ }^{*}(\mathrm{~m})$ & $0.02 / 0.02$ & $0.1 / 0.1$
\end{tabular}

\section{BEAM LUMINOSITY}

Luminosity of the collision of beams with Gaussian distrubution in space is caluculated numerically. Bunch lengths are determined by the RF voltage of the DSR and to be $50 \mathrm{~cm}$ for nucleus beam and $2 \mathrm{~cm}$ for electrons. The beta functions at the interaction points are, $\beta^{*}=10 \mathrm{~cm}$, the technical limit for ions, $\beta *=2 \mathrm{~cm}$ for electrons.

In Table 3, luminosity of e-RI collision is given for typical nuclei which are interested in nuclear physics. General tendency of the change of luminosity with their life times is that the nuclei of which the luminosity $\mathrm{L}>10^{27} / \mathrm{cm}^{2} \mathrm{sec}$, the value corresponds to the minimum luminosity required to measure the charge distribution from the e-RI elastic scattering, have their intrinsic live times longer than about 1 min.

$\underline{\text { Table } 3 \text { Estimated Luminosity for Typical Ions in DSR }}$

Nucleus Intrinsic Lifetime $(\mathrm{sec}) \operatorname{Luminosity}\left(\mathrm{cm}^{-2} \mathrm{sec}^{-1}\right)$

$\begin{array}{lcc}{ }^{6} \mathrm{He} & 0.8 & 1.14 \times 10^{22} \\ { }^{11} \mathrm{Be} & 14 & 1.42 \times 10^{23} \\ { }^{17} \mathrm{~F} & 65 & 6.0 \times 10^{27} \\ { }^{39} \mathrm{Ca} & 0.86 & 1.56 \times 10^{23} \\ { }^{56} \mathrm{Ni} & 5 \times 10^{5} & 1.3 \times 10^{28} \\ { }^{132} \mathrm{Sn} & 40 & 2.4 \times 10^{23}\end{array}$

\section{BEAM-BEAM EFFECT}

Beam-beam interaction is an essentially nonlinear phenomenon, which can results in complicated unstable beam behavior. Influence of the opposite charged beams on each other results in the change of betatron tune of 
particle oscillations. As far as electron beam is much stronger than the ion beam, it is enough for estimations to consider behavior of ion beam in the unchanged field of the electron beam (strong-weak model). For beam-beam interaction the linear part of betatron tune shift $\xi$ is a scale parameter, which defines the strength of beam-beam interactions.

Mechanism of beam-beam instability is still a subject for the accelerator physics, however we obserbed the noise instability in numerical simulations $[6,7]$ where the electron beam size is a subject of noise. The unstable motion of particles appeares when the value of standard deviation in beam-beam kick is changed from turn to turn randomly.

This kind of noise exists naturally in particle-in-cell simulations as well as in real beam due to the mismatch with the channel. Analysis shows that this kind of instability appears in rather simple conditions, where nonlinear beam-beam kick is accompanied with noise. The value of beam-beam kick, required to induce this kind of instability can be as small as $\xi=0.005$, which is typical for experimentally observed beam-beam threshold for ion rings.

\section{CONCLUSIVE REMARKS}

In this paper, typical colliding experiments planned at MUSES project are described. The electron-RI beam collision is a direct way to measure the charge distribution of unstable nuclei. The key issue of this experiment is the available luminosity. It is found that the luminosity can be reached up to $10^{27} / \mathrm{cm}^{2} \mathrm{sec}$ for the nuclei of which the intrinsic life time is $1 \mathrm{~min}$. Larger luminosity is expected for the nuclei beam with longer life time.

To get such a high luminosiyt the important factor of the accelerator aspect is the cooling-stacking of RI beams in the accumulator cooler ring, and the optimized lattice structure at the collider, Double Storage Rings. Also the storage of high current electron beam is a key issue to ovbercome the single bunch and coupled bunch instabilities. Beam-beam effects are simulated on the collision of e-RI beams and it becomes clear that the noise effect on the beam size of electron beam limits the linear tune shift of ions. It should be less than 0.005 which is consistent with the experimentally observed value.

Another experiment of collisoin of RI beams and X-ray produced from the undulator will measure the isotope shift and the hyperfine structure of highly charged radio isotope. This is an atomic process measurement and then we can expect a precise measurement even on the small number of ions. The design of undulator shows that the photon flux of X-ray from the low emittance electron beam will be $10^{18}$ photons $/ \mathrm{sec} \mathrm{mrad}^{2} 0.1 \%$ b.w.
The detailed design of the accelerator and detector system of MUSES project are in progress. The construction of the project is scheduled to start from 2002 fiscal year after the completion of superconducting cyclotron injector.

\section{REFERENCES}

[1] T. Katayama et al., "The MUSES Project at the RIKEN RI beam factory", Nuclear Physics A626(1997) 545.

[2] "MUSES Conceptual Design", to be published as RIKEN publication (1998).

[3] K. Ohtomo and T. Katayama, "Injection Simulation Study at ACR at RI Beam Factory “, to this conference.

[4] I. Watanabe et al., "Electron Cooling at ACR in MUSES Project", to this conference.

[5] T. Takanaka and T. Katayama, "Simulation of the Effects of Longitudinal Broad Band Impedance on an Electron-Cooled Bunched Beam", to this conference.

[6] Y. Batygin and T. Katayama,"Noise Instability in Nonlinear Beam-Beam Interaction", NIM A404(1998)1

[7] Y. Batygin and T. Katayama,"Beam-Beam Instability in Presence of Beam Cooling", to this Conference.

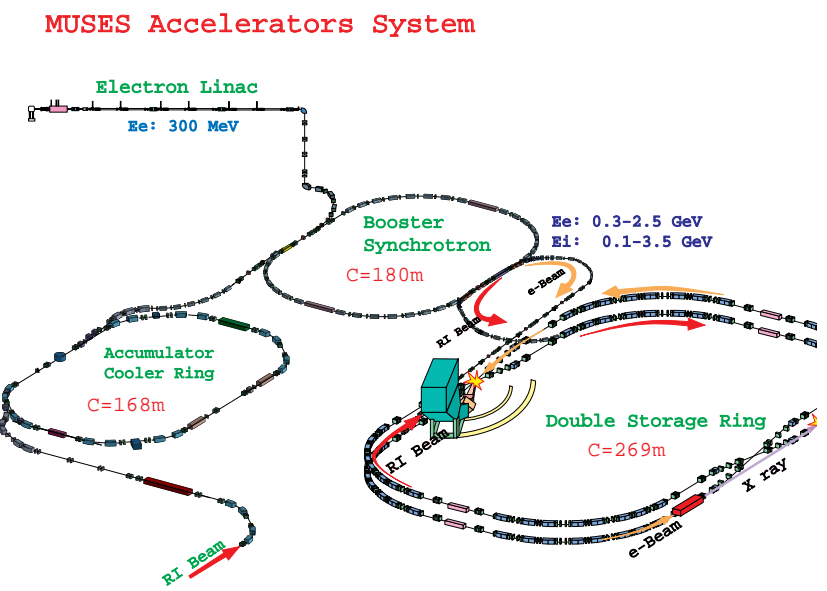

Fig. 1 Scematic Layout of MUSES Accelerators 